\title{
EXPLORING COVID-19 VACCINE ACCEPTANCE AND HESITANCY DETERMINANTS
}

\author{
Tauheed Zahra \\ Ph.D. scholar, Department of Sociology, Quaid-i-Azam University, Islamabad \\ tauheedzahra@gmail.com \\ Farhan Ahmad Faiz \\ Assistant Professor, Department of Sociology, Quaid-i-Azam University, Islamabad \\ farhanfaiz@qau.edu.pk
}

\section{Farrah Ahmed}

Ph.D. Scholar, Department of Sociology, International Islamic University, Islamabad farrahahmed28@gmail.com

\begin{abstract}
The World Health Organization recognizes vaccine related myths and conspiracies as the world's top threat to public health safety, particularly in low middle-income countries. The current study aims to explore the beliefs of the general public towards the vaccine acceptance and the hesitancy. The study explicates the COVID 19 vaccine acceptance and hesitancy determinants through an in-depth qualitative approach. A total of 30 male and female millennials from different education backgrounds were interviewed through an interview guide. This study reveals that people have different beliefs related to the vaccine authenticity which plays a vital role in the reluctance towards it. Findings from paper is similar to literature that people from good educational background have similar thoughts towards COVID 19 vaccination. Disregard for the vaccine was caused by various factors, such as misinformation, safety concerns, and personal knowledge. This level of distrust was associated with the social worlds that participants experienced during the pandemic. COVID-19 vaccine hesitancy is a complex relationship that involves the spread of misinformation. Vaccine programs should provide a focused, localized, and empathetic response to counter misinformation.

Keywords: COVID-19, vaccines, myths, hesitancy, vaccines awareness, pandemic, conspiracy, corona virus

\section{INTRODUCTION}

COVID19 pandemic is the common international issue nowadays and researchers from all over the world are working collaboratively to find out an effective and safe COVID 19 vaccine to prevent this disease(Gostin et al., 2020). From past two years COVID-19 has a heavy disease burden all over the world. According to the World Health Organization Statistics report, the total confirmed cases of COVID19 reached to about 223,449,197of which 4,610,514 deaths were reported till August $31^{\text {st }}, 2020$ in about total 203 countries(Organization, 2020). Therefore, in Pakistan total number of cases were 1.19 million and the associated deaths were 26,947 [4]. The continuous spread of the virus effects the world towards economic crisis. Unfortunately, many of the international health agencies are struggling to acquire the desired targets pertaining to vaccination of the pandemic COVID-19 (Ranasinghe \& Pradeepamali, 2020). Literature has revealed that failure of the health agencies and international organizations to address the concerns of general public offered firm grounds for the development and growth of myths and conspiracy theories regarding the vaccines(Grimes, 2021). This has hampered the vaccine acceptance across the globe with increase in vaccine hesitancy(Aw et al., 2021). In this vein,
\end{abstract}


there is a dire need to promote vaccine acceptance while mitigating the vaccine hesitancy for prolific vaccination outcomes.

Vaccine hesitancy is one of the main factors hampering the effectiveness of the vaccination process of COVID-19. Vaccine hesitancy refers to the people's act of accepting or refusing to take the dose of COVID19 vaccination even if it is available(Lucia et al., 2020). Besides the emergence of marketed vaccines relative safety measures, the response of community towards vaccination is poor indicating vaccine hesitancy. There are numerus factors that are promoting vaccine hesitancy such as political and social gains, social media rumors related to vaccines, exaggeration of negativities pertaining to vaccines, personal background whether medical or non-medical, religious beliefs, and wide spread myths and conspiracy theories along with other related factors(Roozenbeek et al., 2020). Many of the international studies supported the notion that vaccine hesitancy is the major hurdle in acquiring the desired targets of vaccination. Despite of door-to-door service of vaccination and registration of vaccination through an online portal, people were reluctant in taking vaccination(McIntyre et al., 2021). In order to deal with the issue, various campaigns have been conducted to bring in limelight the positive impacts of the vaccination\}\}.Yet, it is observed that even after addressing the safety concerns and measures, the levels of vaccination hesitancy are at an alarming rate as per the weak turnout level on portal(Abu-Farha et al., 2020). Vaccine hesitancy has become a global issue and Pakistan is no exception to it.

Khan et al. (2020) Vaccine hesitancy is not a new phenomenon in Pakistan. It has been observed against the polio vaccine that has resulted in failure to eradicate the disease. The anti-polio campaign when started was resisted through the claims that it is of poor quality, religious scholars prohibited poliovaccine alleging it as an infidel vaccine, dosage recommendations were questioned, and through rumors such as the presence of any active virus inside the vaccination(Kanozia \& Arya, 2021). Similar, reactions have surfaced against the COVID-19 vaccine. With the COVID19 outbreak, rumors were spread all around about the vaccination through the conspiracy theories. Surprisingly, in Pakistan, a famous columnist and political commentator asserts that the COVID19 disease was just an illusion. Influential social actors were of the view that it is conspiracy of Jews to target Muslim regions and to rule all over the world. Furthermore, it was conspired that through 5G towers, they will gain control over the people due to the embedded nano-chips inside their bodies(Islam et al., 2021). In Pakistan, religion card has also been used to refrain people from vaccinating them against the COVID-19 (Chesser et al., 2020). All these aspects significantly promoted vaccine hesitancy among the targeted population. This reveals that there is a dire need to address the issue of vaccine hesitancy to control and prevent the spread of the virus.

It is predicted through literature review that the concept of vaccine hesitancy is known worldwide with its multifactorial background. The widely discussed and encountered issues were the absence of scientific knowledge, ambiguous religious beliefs and safety concerns of public. Different studies also examine the opinion of public about the COVID19 vaccine to assume their level of vaccine hesitancy(Sallam, 2021). It was found that education level, socio-economic status and age are significantly impact the level of vaccine hesitancy among the millennials. The severity and likelihood of vaccine hesitancy in people was also related to lack of knowledge regarding the disease and vaccines, extensive exposure to media generated conspiracies, and social media rumors(Ranasinghe \& Pradeepamali, 2020). Vaccine hesitancy has negatively impacted the social, economic, and health sectors in Pakistan. Therefore, there is need to explore the root cause of vaccine hesitancy in indigenous context to plan better intervention plans. (Troiano \& Nardi, 2021).

The current situation of anti-vaccine beliefs heading towards the dreadful outcomes, promoting the non-acceptance of the vaccine of COVID 19 (Bastani \& Bahrami, 2020). WHO identified different types of myths and conspiracies as a world top threat towards the public health safety, specifically in the developing countries like India, Pakistan. Bangladesh etc.(Moody et al., 2010). It was found that vaccine acceptance is extremely low in the developing countries irrespective of their social backgrounds, profession, and educational level. The irrational beliefs regarding the international vaccines are deeply inculcated among the targeted population ||/. In order to promote the vaccine acceptance, World Health Organization (WHO) is taking strict measures to detect the conspiracies and myths associated with the 
Exploring COVID 19 Vaccine Acceptance and Hesitancy Determinants

disease and vaccines to eradicate them(Nishimura et al., 2021). Literature reveals that vaccine acceptance $\mathrm{n}$ the developing nations could be enhanced by rationally addressing the religious/spiritual beliefs, offering authentic information regarding the vaccination process, dealing with the vaccine misconceptions and misinformation. International health organizations are investing huge efforts and resources to mitigate the anti-vaccine notions to boost up the vaccine acceptance (Organization, 2020). In this vein, media could play an influential role as evident in the past literature.

Media is used as a mediator to discuss all such theories by the community of Pakistan. For instance, Pakistan ex-foreign minister criticized United States and alleged that they are the creator of this virus in the state from where it will slightly move to China and then to rest of the world. Media is the primary source of information for majority of the targeted population The information disseminated by the media platforms are internalized by the audience. Literature reveals that media play critical role in building opinion of the audience. In reference to COVID-19 vaccine, social media has offered grounds for spreading irrational beliefs regarding the vaccination. Religious grounds were also used to influence the audience. Such conspiracies and discussions further act as a barrier on the path of COVID19 vaccination programs success, eventually leading to a greater level of vaccine hesitancy(Khan et al., 2020b). Align with these facts, WHO highlighted that it's not only the pandemic that we have to deal with but also infodemic.

This study was conducted with the primary objective to access the effect of educational background and work field on responses towards COVID-19 vaccine hesitancy. The secondary objective is to identify the most encouraging and discouraging factors towards vaccine hesitancy and how significant is the role of social media in shaping the attitudes of people towards its acceptance.

\section{Primary objective}

The primary objective of the study is to check the effect of educational background and work field on responses towards vaccination.

\section{Secondary objective}

The secondary objective is to identify the most encouraging and discouraging factors to take the vaccine hesitancy and how significant is the role of social media in shaping the attitudes of people towards acceptance.

\section{REVIEW OF LITERATURE}

The epidemic of disease is referred as a pandemic that has gained immense worldwide attention due to its global spread. The global proportions of COVID19 disease has created a panic everywhere and reached at a label of pandemic(Organization, 2020). WHO Country office reported about the virus for the first time on December 31 $1^{\text {st }}, 2019$ in Wuhan, China, that was first detected in the form of pneumonia. In the earlier times, the causative agent of this virus was unable to identify, thus, it was classified as pneumonia that belongs to corona virus (CoV) family, is novel and of unknown etiology. The corona virus disease 2019 abbreviated as COVID19 has global reach due to its highly contagious nature. According to the World Health Organization Statistics report, the total confirmed cases of COVID19 reached to about 7,54,948 out of which 36,571 deaths were reported till March $31^{\text {st }}, 2020$ in about total 203 countries(Organization, 2020).

The rate of fatalities of this virus are very high as per the Centers for Disease Control and Prevention (CDC) preliminary data. The most frequent deaths were reported with the patients having any underlying health issues or the older adults as their immune system is already weak. Furthermore, high mortalities were also a result of absence of a vaccine, no proper effective medical treatment and other such reasons. Study reveals that for the improvement of public response, it is important to share scientific knowledge rapidly that is a key to improve public health(Song \& Karako, 2020). This could include the information provided by the epidemiologists through real time experiences, management of patients by the clinicians and modelers assisting in the effective interventions and for comprehending the future 
developments. Media and other official authorities of public health poses great attention to this virus as it halts businesses, shut down businesses, shutter schools and the whole system is shaken through this disease spread that needs to be controlled(Chesser et al., 2020). The effective management of COVID19 crisis is under debate such as by the United States through closing the borders and regulating travel. These measures depict public concern about health safety as (Chesser et al., 2020)

The demand of vaccine and its acceptance by the public is a complicated process that is even context-specific, this process is dependent and varies as per the community perceived behavior, place, and time. For instance, the vaccine acceptance in China is predicted through the perceptions of public and the demographics(Sallam, 2021). The vaccine of the seasonal influenza is avoided in Ireland due to the lack of their trust, misconceptions about the vaccine effectiveness and other such reasons. Moreover, the acceptance of vaccine in United states for Influenza is due to their health insurance, social influence, and influenza vaccine perceived effectiveness. At the same time, it is also reported that the higher level of vaccine uptake is due to a greater level of confidence in vaccination and the lower uptake of vaccine is associated with the greater hesitancy in US. Furthermore, the vaccine acceptancy in Hong Kong is dependent upon the history of vaccination and the anxiety level(Lin et al., 2021) . The report about the parental attitudes in the United Arab Emirates about the vaccination of their children indicates that the vaccine hesitancy is only present in $12 \%$ of the parents. The factors that are involved in vaccine hesitancy includes the side effects about $35 \%$, vaccine safety to about $17 \%$ and injections to about $28 \%$. Moreover, the intention of people to get vaccination is influenced by their history of seasonal flue vaccination(Sallam, 2021).

The vaccine uptake depends upon the trust of public and is relatively different in medium income settings than low income settings. Literature also states that the willingness of a general public to decline or accept a particular vaccine such as H1N1 depends upon various factors(Mesch \& Schwirian, 2015). These factors may include the event severity, ethnicity, personal consequences, infection perceived risk, and any previous history of vaccination. Furthermore, the different behavior oh human in different population groups make the equitable vaccination process complicated an which could vary in time and space. The vaccine hesitancy is discussed in many different aspects like some authors discussed it as per the "theory of planned behavior" that is the behavioral health model. Furthermore, the uptake of vaccination could be increased through the policies and tailored interventions in this regard(Shmueli, 2021).

The acceptance of COVID19 vaccination as well as its determinants are widely researched to make it possible. In China, a study was conducted on their healthcare workers that showed a relative increase in their good intentions to take vaccine shot as compared to the local public. Moreover, it is reported that the COVID19 vaccination is declined by only $20 \%$ of the total population in United States. Thus, it is declared that the acceptance of vaccination is rather context specific and it can only vary with the changes in the culture, geography, or the sociodemographic differences(Yin et al., 2021).

The fear of vaccination is also elevated through media that even fought with the effectiveness of vaccine and its evidences. The vaccine hesitancy is influenced through media, causing detrimental impact on the people perceptions about vaccination. The 2009 National Immunization Survey confirmed the finding that internet spread false rumors, misinformation, myths about vaccination leading to the vaccine hesitancy in people or delayed acceptance(Mesch \& Schwirian, 2015).

It is important here to notice that the "infodemic" term can also be used for the COVID19 pandemic. The misinformation about COVID19 crisis is fueled by the uncertainty, fear and the conspiracies claims about virus in different populations. These type of conspiracy theories promote vaccination hesitancy such as the bizarre hoax. This concept about the microchips inside human body to track human actions indulged through vaccination create falsified information. There are other allegations like the virus is human generated and its vaccination could lead to infertility limit the participation of female in getting vaccination(Nielsen et al., 2020).

In the social media technological era in which we are living, the greatest pandemic risk is the viral misinformation as this could lead to the sustainability of health issues. The information about the pandemic should not be dependent upon the social media platforms, instead people should be well aware, 
Exploring COVID 19 Vaccine Acceptance and Hesitancy Determinants

educated, have updated information about facts and figures. This could then help in reducing the level of vaccine hesitancy.

\section{METHODOLOGY}

The exploratory descriptive qualitative design of research study was used to explore the research idea of this study. The research design in which there are limited resources and time, is relevant and that extract the information from the phenomenon experienced through investigation is referred as an exploratory descriptive qualitative design. This type of research design provides an in-depth information about the idea under research. The exploratory design used in this research as per (Moody et al., 2010)is an approach that investigates a problem from its preliminary step and distant from its saturation point. This research design is preferred as it is suitable in cases where there is an efficient and relevant solution is required through solving the problem's exact nature. This research design do not rely on the previous observations about the research topic under study but also investigates the root causes of a problem. The COVID19 pandemic has influenced different areas, the effect on education system transformation is already well researched. However, the phenomenon about the effect of COVID19 outbreak on the job roles of administrative heads and principal needs to be further explored. As the area under research is not well explored, thus, exploratory research design is selected that will provide ground to the researchers for their analysis. Furthermore, this research has a flexible nature which during the whole process of research could adapt necessary changes. The first-hand information attained through the exploratory research help the researcher in finding out the real worth of its investigation as well as the significance of the chosen topic. These positive points about the exploratory research convinces to use this approach.

The qualitative research design is used for research that according to (Pathak et al., 2013)is mostly used textual or numerical data for examining the opinions, concepts and experiences of people. It is also used to explore new domains that are not researched till then. This research approach brings into spotlight new areas of research and fill the literature gaps. For this purpose, the qualitative research approach is selected to gather information and to investigate on the determinants of corona virus vaccine hesitancy and acceptance(Gelling, 2015).

The data used in this research was collected through qualitative research approach having interview sessions with both male as well as female. These respondents are from different work fields and educational backgrounds. The open ended questions were used in the interviews and for designing the questions, theoretical framework and literature review was used. The questions used in the interview were easy to understand, non-leading, non-threatening, and unbiased. According to (Rowley, 2012) the open ended questions help respondents to explore the topic of interest by opening up their mind. The data collection process is eased through the beforehand preparation of an interview guide. For this study, interviews were conducted from total 30 respondents.

The common patterns of response are identified through a thematic analysis done on the data collected through qualitative approach and interviews. In the academic settings, data analysis technique that is commonly used and is essential is the thematic analysis. According to (Braun \& Clarke, 2014) for the thematic analysis, limited resources are required and it is a valid and reliable technique to be used. To address the objectives of research in a comprehensive manner, it is important to convert the qualitative data in large amount into the responses that are meaningful. In this study case, the hindrances in the use and applications of COVID19 vaccination could be identified as well as the relevant myths recognized through a thematic analysis. The important and appropriate required information about the research questions will be gathered through the interview process.

\section{MAJOR FINDINGS}

Understanding the perception of people about COVID 19 vaccination and its acceptance in their lives. People from different backgrounds and educational sectors are part of this study. To meet the purposes of the study in-depth interviews were conducted using interview guide made by the researcher. None of the participants had ever tested positive for COVID-19. Some of them suspected they might have contracted the virus earlier than February or March. Most of them showed reluctance to vaccinate due to their own 
reasons, two of my respondents out of 30 are vaccinated. The remaining three quarters of respondents had friends, family members, colleagues or neighbors who had tested positive for COVID-19 and experienced various symptoms and outcomes from being mildly ill to hospitalization and even death. Finding out that someone they were close to had been very ill with COVID-19 did appear to make the virus more 'real'. This section deals with thematic analysis of provided data. Thematic analysis is the multi-phased process. Transcription of the spoken words is the first step in thematic analysis (Braun \& Clarke, 2014) Major themes in result of thematic analysis were factors including false information responsible for vaccine hesitancy false information, lack of sufficient and transparent information, lack of trust in public and government agencies, informal media sources and vaccine information, role of negative belief and faiths, Myths and conspiracy theories on COVID-19 existence, vaccination and its consequences.

\section{Factors including false information responsible for vaccine hesitancy}

It is known through research that the misinformation about corona virus disease and the conspiracy theories are widely present. These information platforms mislead the social group and restrict them from using vaccine for resolving the health burden(Sohail et al., 2021). The perceptions of a common man are extensively influenced by the role of media, the conspiracy theories that are far-fetched elevates the vaccine hesitancy level among people and raise the anti-vaccine groups. For instance (Y. H. Khan et al., 2020a) reported that the similar kind of conspiracy theories as well as anti-vaccine groups emerged during the polio virus outbreak and failed Pakistan to get rid of polio completely. Therefore, the risk of corona virus is also widespread issue in Pakistan.

As one of my respondents says that: "Its just a western agenda to lessen the population of Muslim countries and firstly they create it and now selling their vaccines and earning money."

Another said that: "It's just a game and corona virus doesn't exsits bs karobar chamka raye han jis main sab shamil han pharmaceuticals bhi aur doctors bhi".

A respondent judge by profession he says "prevention is better than cure I will wear mask but I will never go for vaccination "they are making money just by making people fool yeh saray to pagal han"

One of my female respondent says that the "lady health worker living near my house suggest me not to take it yet because you have to raise your family and you are too young it may cause infertility or the child become impaired"

"my doctor prescribed me not to take the second shot because I am pregnant now. She said it might cause illness to the child so better not to take it"

"I am struggling to have a baby I visited the gynecologist she advised me not to take vaccination if you want to conceive because vaccination will cause problem in conceiving"

"I am nikahfied and my mother and my mother-in-law both don't allow me and my wife to take the vaccination because they think that it's not good for the ones who want to start their family so we both haven't take it as we are so concerned about our future life"

There are some believers who claimed that vaccine itself is the reason behind coronavirus. Few respondents reported that the genes are permanently altered, and the DNA is damaged through vaccination. Moreover, some believe that it contains the chips that are incorporated in the body of a vaccinated person to monitor its activities for easy targeting. The misinformation spread in the environment about the spread of coronavirus make individuals reluctant to get vaccinated due to the fear of getting health issues, safety concerns and others. Individual's both male and female have fear of taking the shots.

\section{Lack of sufficient and transparent information}

The transparency of information is another rapid issue as it is not ensured and the wrong information creates misunderstanding leading to vaccine hesitancy among public. The information about the efficacy of vaccine and its safety should be correct, accessible, and transparent for all. The unauthentic data about the vaccination make people presume about vaccine in a negative way making them reluctant to get their shots especially through watching the social media sites information. Respondent claims that 
"they don't even test this vaccination either it will prevent the disease or not; the western countries send free vaccination into Pakistan just because they want to end up the Muslim nation. Israel is behind all that because they are our biggest enemy"

One of my respondent who is in medical field and working as a nurse she said: "I actually don't want to take this because I know the reality of this vaccination, our hospital makes it mandatory that's why I got vaccinated otherwise they will not release my pay"

"another respondent who is doctor by profession she said I think this vaccination is of no use that's why I would prefer not to take it... I don't have any false beliefs about it but I don't think it works. It's better to just wear masks and use sanitizer"

The aforementioned account confirms that the urgency of situation also envisaged the doubts about the vaccine safety and its usefulness. (Kelen \& Maragakis, 2021) states that the prior to the launch of vaccination, the insufficient data, unreliable records, rushed testing and unavailability of enough information makes the people associated with the medical field reluctant to get vaccinated. The people do not get vaccinated sometimes even if they are willing to as they are less aware about the eligibility to get a vaccine shot. Some authors such as (Jain et al., 2021) and (Mahmud et al., 2021) indicates that the lack of information or misinformation about the vaccine side effects also restrain people from getting vaccinated. One respondent claim that: "my father is not a heart patient but after having vaccination his blood pressure use to high later when he visited doctor he was diagnosed with the heart disease"

\footnotetext{
"my uncle is perfect and he worked in Dubai he came to Pakistan got vaccinated and after two days of vaccination he died, he doesn't have any medical history he is too young as well as my aunt she is a bit old but she also doesn't have any disease she also died after getting vaccination. So I would never ever get vaccinated neither any of our family member after these incidents"

"they didn't even bother to take medical history before vaccination. I was amazed when I went for my mother's vaccination, she is a blood pressure patient, I told them about my mother's health and one of them said ok fill that in form...I took my mother back because I am afraid how will it affect my mother"
}

Jain et al. (2021) Since the vaccines were only available for a few months, people were already worried that they could cause long-term effects. This is also due to the lack of information about their effects. Another reason why people are reluctant to get vaccines is that they tend to believe that they cause-and-effect theory. The media reports about deaths and major illness outbreaks linked to coronavirus vaccines have made people afraid to get vaccinated. Despite the evidence that vaccines do not cause deaths, people were still afraid to get vaccinated despite the assurance that they could prevent illness(Kanozia \& Arya, 2021). In this study, the authors found that the non-consenting nature of the medical students when it comes to providing vaccine registration data is a contributing factor to their reluctance to use the vaccine. Due to the lack of reliable information on the efficacy and safety of vaccines, many people who rely on them for their medical needs have been reluctant to vaccinate (Raghavan, P. 2021, April 13).

\section{Lack of trust in government and public agencies}

"respondant explain that yeh pani dal kay vaccine laga raye han aur pagal bna raye, khana to ghareeb admi tak pohncha ni saktay vaccine kia lgain gay. Yeh to pagal han humaray upar test kar raye han aur yeh logn ko lgaye ja raye" 
"one respondent says that the vaccination which government is providing free is not good for health, they are testing on us may be the one which is expensive so called rich people can afford is the real one"

When faced with a situation that is unexplainable, people are always apprehensive of their governments. It was also observed that the public's distrust of government and the public authorities led to the development of vaccines (Hashim, 2021). The development of coronavirus vaccines for emergency use caught the public's attention. Some conspiracy theorists believed that the government was involved in the vaccine's development. The authors of this study stated that political affiliation and context can influence the approval of vaccine(Bokemper et al., 2021). They also noted that people's understanding of the vaccine's efficacy and safety can be influenced by public figures and political parties (e.g. Dr. Anthony Fauci) who are non-partisan(Fauci et al., 2005).

The vaccine hesitancy as well as acceptance is dependent upon people's belief, the fear of any side effects, safety of vaccination, trust of people in the vaccination system, absence of good recommendations from healthcare workers and health relevant issues caused by vaccination. Importantly, it is stated that the vaccine effectiveness and its benefits are associated with the beliefs that affect the vaccine hesitancy and acceptance. For instance, the family's attitude especially that of parents towards getting vaccinated, friends conversations, wariness of the motives behind getting vaccinated greatly influence the decision of people about vaccination (Moody et al., 2010).

\section{Formal, informal media sources and vaccination information}

Besides the vaccination effectiveness and fortification evidences, the fear of vaccination is intensified by the media that is one of the major player. The vaccine hesitancy is enormously affected with the facts shared on media about the vaccination. These controversies about the safety of vaccine upsurge the antivaccine community behavior as well as vaccine hesitancy through the circulation of popular articles, talk shows and news headlines.

"whenever I opened any social media website I saw different news related to vaccination. I have a very close friend who's brother is vaccinated but he died later so after seeing realities, I found these media message real and I have strong believe in that"

People have different faiths and beliefs on their own, the right wing community thinks that vaccination contain haram elements like pork or it contain any animal cells from the aborted fetus(Ahsan et al., 2021).

The answers attained through the interview questions were related to our literature findings. The answers also assert that the misinformation or the misleading videos, images and messages are viral on internet as well as social media platform. Literature also confirms the findings such as (Y. H. Khan et al., 2020b) affirms that the point of view of Pakistan's well-renowned public figure about coronavirus are a support to vaccine hesitancy as he called it a conspiracy by the non-Muslim.

Respondent says that: "it's just a propaganda of social media owners as well as the non-Muslim nations just to practice absolute control on the population of whole world as well as use them for profit generating",

"bemari aur shifa ALLAH kay hath main ha insan ko tadbeer karni chahye aur yeh azmaish ha aur ALLAH is lye koi hal bhi nikalay ga"

Vaccination refusal is often linked to moral beliefs and philosophical beliefs regarding the importance of health immunity. It has also been associated with religious beliefs. In 2011, anti-vaccine propaganda intensified its attacks on the use of vaccine. It claimed that the vaccine is made up of gelatin that is haram and is forbidden in Islam(Areas, 2011).

\section{Myths and conspiracy theories on COVID-19 existence, vaccination and its consequences}

"sometimes I believe sometimes I don't, when I went to hospital for a checkup I believe in the existence of COVID while watching the behaviors of hospital administration. But when it comes to gatherings such as PDM rallies, religious sermons, lavish marriage ceremonies, parties and others, I am unable to decide whether COVID exist or not. I believe when government want money or foreign aid they started says that the new wave of corona cases 
arises and later they use all money for their heinous political motives, those translucent policies are the main hindrance for me to believe in COVID existence as well as the impact of vaccination"

"researcher says that I got vaccination because all my family got it, I don't have firm belief that it will prevent me from corona I was just taken because everyone is taking. Believe me sometimes I think that corona is a myth"

Some individuals do not believe in COVID19 existence and are not very comfortable with the concept of a flu-like illness. Some people believe that COVID-19 is just a business that's being done by health care workers to collect COVID-19 benefits. Others think that doctors are intentionally diagnosing COVID-19 to get paid.

\section{Cultural substitutes for COVID vaccine}

"In the start of COVID 19 all of my family members were positive we take a lot of yakhni and natural fruits and we are ok and sana maki kehwa helps us a lot to recover"

"I do believe in COVID but I dnt believe in vaccination.. my brother is a doctor and its mandatory for them to take vaccine but he adviced us not to take it just wear masks eat healthy food protect youself from big gatherings that's all. We see them as a medical practioner not in favor of vaccine how would we go for it"

The goal is to develop a vaccine that will be effective enough to end the pandemic caused by the COVID-19 virus. The vaccine's effectiveness is relative to the population's acceptance of it. In Australia, $85.8 \%$ of the respondents would accept the COVID-19 vaccines if they were offered. In the US, only $67 \%$ would accept the vaccine(Abu Farha et al., 2021).

\section{DISCUSSION}

As the COVID-19 pandemic overwhelms health systems in the developed countries, Pakistan has been spared from the worst of its high mortality. Its young population and adherence to social distancing mandates have helped lower its cases relative to the developed nations. The study revealed that the participants were exposed to a wide variety of misinformation, which often came from social formal and informal media sources. This misinformation led to confusion and distress, which could affect their confidence in the vaccines' safety. It has been known that misinformation thrives during times of uncertainty and anxiety, and COVID-19 provided a perfect breeding ground. This study shows that people tend to believe that conflicting information makes them more anxious, which in turn increases their willingness to believe it. In February 2020, the WHO stated that the outbreak of COVID-19 had been accompanied by a massive misinformation campaign. Despite the lack of sufficient evidence to measure how far-reaching and influential the misinformation was, it has been known that it affected the willingness of people to get vaccinated. In our study, we found that misinformation about COVID-19 was spreading in Pakistan, which has affected the lives of participants. The level of confusion and distrust experienced by those who were most likely to be affected by the vaccine has a negative effect on their proclivity towards uptake. COVID-19 has affected various aspects of people's lives. One of the most prevalent discussions is the prevention of infection through the COVID-19 vaccine.

Working professionals, young adults, and housewives rely on social media platforms to get informed about the COVID-19 vaccine. Their dependence on these sources may be related to the quality of information available to them. Contrary to this, hospital-based groups had better understanding of the COVID-19 vaccine but in our study few of them show reluctance towards vaccination just because of its authenticity as it was developed and marketed in less time. Its use was also promoted through various social media platforms. We found that the level of knowledge about COVID-19 vaccine did not correlate with a positive perception among the general population. Vaccine acceptance was achieved by factors such as the perceived safety of vaccines, the freedom to vaccinate, and the lack of risk in vaccinations. Participants also felt that they could trust the information about the vaccine. Moreover, many of the participants believed that vaccination is a propaganda against the Muslims and the non-Muslims tend to rule the world. COVID 19 vaccination would cause infertility in males and women think that they are not 
able to produce children or it may cause complication is the most common myth among the respondents. Many of them have perception that the vaccination which is not free is authentic as compared to vaccine available for general public. The main factors that led to the rise in vaccine hesitancy were the perception of safety, efficacy, and misinformation about the vaccine. Other factors include government conspiracies and faulty vaccines.

\section{CONCLUSIONS}

The results of this study revealed that, the misinformation from different sources leads to hesitancy towards COVID-19 vaccination among the general public of Pakistan. Despite of different educational and social backgrounds people have strong faith in misinformation related to vaccination, they do believe in the existence of virus but false perception about vaccination is major threat to the upcoming immunization program for the control of COVID-19 in Pakistan.

\section{RECOMMENDATIONS}

Local authorities can counter misinformation through targeted local responses. This can be done through the establishment of targeted local responses and the monitoring of social media outlets for spreading disinformation. It is clear that the anxiety around the COVID-19 vaccine is rooted in misinformation, and it is essential that we provide factual information that is sensitive and non-judgmental. The public's willingness to accept a given vaccine depends on the information and sentiment surrounding it. This is why the government should engage social media outlets to provide accurate and balanced information. In addition, celebrity endorsements and many other social media platforms can motivate people to get the vaccine. This information should be made public in a way that is understandable to the public. Public health authorities should also handle these issues.

A communication helpline should be generated for the help of general population to overcome their fears related to vaccination. Awareness should be spread by the help of different media platforms as well as the religious leaders about the importance of vaccination. Policymakers and officials need to help in making different strategies to address the major concerns of vaccine-resistant groups. Scientists and medical experts should also share their knowledge, efficacy and effect of virus on human body and importance of vaccination. Only a collaborative effort between government institutions, academicians, scientists, and journalists can get rid of this hesitancy towards the vaccine, which latterly helps to survive this pandemic. This study can be utilized by healthcare professionals and government agencies to develop effective and relevant messages related to COVID-19 vaccine to strengthen the positive attitude of the public.

\section{REFERENCES}

Abu Farha, R. K., Alzoubi, K. H., Khabour, O. F., \& Alfaqih, M. A. (2021). Exploring perception and hesitancy toward COVID-19 vaccine: A study from Jordan. Human Vaccines \& Immunotherapeutics, 1-6.

Abu-Farha, R. K., Alzoubi, K. H., \& Khabour, O. F. (2020). Public willingness to participate in COVID19 vaccine clinical trials: A study from Jordan. Patient Preference and Adherence, 14, 2451.

Ahsan, M., Based, M., Haider, J., \& Kowalski, M. (2021). COVID-19 detection from chest X-ray images using feature fusion and deep learning. Sensors, 21(4), 1480.

Areas, F. A. T. (2011). Federally Administered Tribal Areas. Population, 1.

Aw, J., Seng, J. J. B., Seah, S. S. Y., \& Low, L. L. (2021). COVID-19 vaccine hesitancy-A scoping review of literature in high-income countries. Vaccines, 9(8), 900.

Bastani, P., \& Bahrami, M. A. (2020). COVID-19 related misinformation on social media: A qualitative study from Iran. Journal of Medical Internet Research.

Bokemper, S. E., Huber, G. A., Gerber, A. S., James, E. K., \& Omer, S. B. (2021). Timing of COVID-19 vaccine approval and endorsement by public figures. Vaccine, 39(5), 825-829. 
Exploring COVID 19 Vaccine Acceptance and Hesitancy Determinants

Braun, V., \& Clarke, V. (2014). What can "thematic analysis" offer health and wellbeing researchers? Taylor \& Francis.

Chesser, A., Drassen Ham, A., \& Keene Woods, N. (2020). Assessment of COVID-19 knowledge among university students: Implications for future risk communication strategies. Health Education \& Behavior, 47(4), 540-543.

Fauci, A. S., Touchette, N. A., \& Folkers, G. K. (2005). Emerging infectious diseases: A 10-year perspective from the National Institute of Allergy and Infectious Diseases. International Journal of Risk \& Safety in Medicine, 17(3, 4), 157-167.

Gelling, L. (2015). Qualitative research. Nursing Standard (2014+), 29(30), 43.

Gostin, L. O., Karim, S. A., \& Mason Meier, B. (2020). Facilitating access to a COVID-19 vaccine through global health law. The Journal of Law, Medicine \& Ethics, 48(3), 622-626.

Grimes, D. R. (2021). Medical disinformation and the unviable nature of COVID-19 conspiracy theories. Plos One, 16(3), e0245900.

Islam, M. S., Kamal, A.-H. M., Kabir, A., Southern, D. L., Khan, S. H., Hasan, S. M., Sarkar, T., Sharmin, S., Das, S., \& Roy, T. (2021). COVID-19 vaccine rumors and conspiracy theories: The need for cognitive inoculation against misinformation to improve vaccine adherence. PloS One, 16(5), e0251605.

Kanozia, R., \& Arya, R. (2021). "Fake news", religion, and COVID-19 vaccine hesitancy in India, Pakistan, and Bangladesh. Media Asia, 1-9.

Kelen, G. D., \& Maragakis, L. L. (2021). COVID-19 Vaccines: Myth Versus Fact. Enlace: Https://Www. Hopkinsmedicine. Org/Health/Conditions-and-Diseases/Coronavirus/Covid-19-Vaccines-Mythversus-Fact.

Khan, Y. H., Mallhi, T. H., Alotaibi, N. H., Alzarea, A. I., Alanazi, A. S., Tanveer, N., \& Hashmi, F. K. (2020a). Threat of COVID-19 vaccine hesitancy in Pakistan: The need for measures to neutralize misleading narratives. The American Journal of Tropical Medicine and Hygiene, 103(2), 603.

Khan, Y. H., Mallhi, T. H., Alotaibi, N. H., Alzarea, A. I., Alanazi, A. S., Tanveer, N., \& Hashmi, F. K. (2020b). Threat of COVID-19 vaccine hesitancy in Pakistan: The need for measures to neutralize misleading narratives. The American Journal of Tropical Medicine and Hygiene, 103(2), 603.

Lin, C., Tu, P., \& Beitsch, L. M. (2021). Confidence and receptivity for COVID-19 vaccines: A rapid systematic review. Vaccines, 9(1), 16.

Lucia, V. C., Kelekar, A., \& Afonso, N. M. (2020). COVID-19 vaccine hesitancy among medical students. Journal of Public Health (Oxford, England).

Mahmud, M. S., Talukder, M. U., \& Rahman, S. M. (2021). Does 'Fear of COVID-19'trigger future career anxiety? An empirical investigation considering depression from COVID-19 as a mediator. The International Journal of Social Psychiatry, 67(1), 35.

McIntyre, P., Joo, Y. J., Chiu, C., Flanagan, K., \& Macartney, K. (2021). COVID-19 vaccines-are we there yet? Australian Prescriber, 44(1), 19.

Mesch, G. S., \& Schwirian, K. P. (2015). Social and political determinants of vaccine hesitancy: Lessons learned from the H1N1 pandemic of 2009-2010. American Journal of Infection Control, 43(11), $1161-1165$.

Moody, W., Kinderman, P., \& Sinha, P. (2010). An exploratory study: Relationships between trying on clothing, mood, emotion, personality and clothing preference. Journal of Fashion Marketing and Management: An International Journal.

Nielsen, R. K., Fletcher, R., Newman, N., Brennen, J. S., \& Howard, P. N. (2020). Navigating the 'infodemic': How people in six countries access and rate news and information about coronavirus. Reuters Institute.

Nishimura, Y., Miyoshi, T., Hagiya, H., Kosaki, Y., \& Otsuka, F. (2021). Burnout of healthcare workers amid the covid-19 pandemic: A Japanese cross-sectional survey. International Journal of Environmental Research and Public Health, 18(5), 2434.

Organization, W. H. (2020). World Health Organization coronavirus disease (COVID-19) dashboard. World Health Organization. 
Pathak, V., Jena, B., \& Kalra, S. (2013). Qualitative research. Perspectives in Clinical Research, 4(3).

Ranasinghe, J., \& Pradeepamali, J. (2020). After corona (COVID-19) impacts on global poverty and recovery of tourism based service economies: An appraisal.

Roozenbeek, J., Schneider, C. R., Dryhurst, S., Kerr, J., Freeman, A. L., Recchia, G., Van Der Bles, A. M., \& Van Der Linden, S. (2020). Susceptibility to misinformation about COVID-19 around the world. Royal Society Open Science, 7(10), 201199.

Rowley, J. (2012). Conducting research interviews. Management Research Review.

Sallam, M. (2021). COVID-19 vaccine hesitancy worldwide: A concise systematic review of vaccine acceptance rates. Vaccines, 9(2), 160.

Shmueli, L. (2021). Predicting intention to receive COVID-19 vaccine among the general population using the health belief model and the theory of planned behavior model. BMC Public Health, 2l(1), 1-13.

Sohail, M. U., Munir, H., Aslam, O., \& Hayat, K. (2021). Covid-19 Vaccination in Pakistan: Challenges and Priorities. Ilkogretim Online, 20(5).

Song, P., \& Karako, T. (2020). COVID-19: Real-time dissemination of scientific information to fight a public health emergency of international concern. Bioscience Trends.

Troiano, G., \& Nardi, A. (2021). Vaccine hesitancy in the era of COVID-19. Public Health.

Yin, F., Wu, Z., Xia, X., Ji, M., Wang, Y., \& Hu, Z. (2021). Unfolding the determinants of COVID-19 vaccine acceptance in China. Journal of Medical Internet Research, 23(1), e26089. 